\title{
LINEAR SYSTEMS OF REAL QUADRATIC FORMS. II
}

\author{
EUGENIO CALABI ${ }^{1}$
}

\begin{abstract}
Consider a mapping $F: \mathbf{R}^{n} \rightarrow \mathbf{R}^{3}(n>3)$ defined by an ordered triple of real-valued quadratic forms; if some linear combination of these three quadratic forms is positive definite, then the image set under the map is a convex cone.
\end{abstract}

This note is a sequel to an earlier one [1] with the same title, dealing with maps of $\mathbf{R}^{n}$ into $\mathbf{R}^{k}$ defined by $k$ quadratic forms in $n$ real variables. One of the problems to which this note is devoted is that of describing properties of the image set under such a mapping, especially when $n \geqslant k$.

We begin with a few elementary remarks.

1. A set of $k$ given quadratic forms is linearly dependent, if and only if the image set under the mapping that they define into $\mathbf{R}^{k}$ lies in a vector subspace, so that it is of ten convenient to assume without loss of generality that the $k$ given quadratic forms are linearly independent.

2. If $F: \mathbf{R}^{n} \rightarrow \mathbf{R}^{k}$ is defined by $k$ quadratic forms $u_{\alpha}=f_{\alpha}(x)\left(x \in \mathbf{R}^{n} ; \alpha=\right.$ $1,2, \ldots, k)$, then the image set $F\left(\mathbf{R}^{n}\right) \subset \mathbf{R}^{k}$ is always a cone, that is to say a set of points (vectors) in $\mathbf{R}^{k}$ closed under multiplication by nonnegative scalars. This set lies in a closed half-space, defined, let us say, by a homogeneous linear inequality

$$
\sum_{\alpha=1}^{k} c_{\alpha} u_{\alpha} \geqslant 0 \quad\left(\left(c_{1}, \ldots, c_{k}\right) \neq(0,0, \ldots, 0)\right)
$$

if and only if the quadratic form

$$
f_{c}=\sum_{\alpha=1}^{k} c_{\alpha} f_{\alpha}
$$

is positive semidefinite.

3. If, for some real constants $\left(c_{1}, \ldots, c_{k}\right)$ the quadratic form (1) is positive definite, and if $n<\infty$, then the mapping $F: \mathbf{R}^{n} \rightarrow \mathbf{R}^{k}$ defined by $u_{\alpha}=f_{\alpha}(x)$ $(1 \leqslant \alpha \leqslant k)$ is a proper map into the half-space $\{0\} \cup\left\{u=\left(u_{\alpha}\right) \in \mathbf{R}^{k} \mid \sum_{\alpha=1}^{k} c_{\alpha} u_{\alpha}\right.$ $>0\}$.

Our main result is the following one.

TheOREM. If $F: \mathbf{R}^{n} \rightarrow \mathbf{R}^{k}$ is defined by $k$ quadratic forms $u_{\alpha}=f_{\alpha}(x)\left(x \in \mathbf{R}^{n}, \alpha=\right.$ $1,2, \ldots, k)$ and satisfies the following assumptions:

(a) $n>k=3$.

(b) For a certain set of $k$ constants $c_{1}, \ldots, c_{k}$ the quadratic form $f_{c}=\sum_{\alpha=1}^{k} c_{\alpha} f_{\alpha}$ on $\mathbf{R}^{n}$ is positive definite. Then the image cone $K=F\left(\mathbf{R}^{n}\right)$ in $\mathbf{R}^{k}$ is a convex cone.

Received by the editors April 10, 1981.

1980 Mathematics Subject Classification. Primary 15A63; Secondary 52A15.

1 Supported in part by NSF Grant \#MCS 78-02285. 
PROOF. It follows from Remarks 2 and 3 above that the image cone $K=F\left(\mathbf{R}^{n}\right)$ satisfies at least the following properties:

(a) $K$ is contained in the closed half-space $\bar{H}_{c}$ defined by the linear inequality $\sum_{\alpha=1}^{k} c_{\alpha} u_{\alpha} \geqslant 0$.

(b) If $n<\infty$, then the map $F$ is a proper map; indeed there is constant $\varepsilon>0$ such that, for all $x \in \mathbf{R}^{n}, \sum_{\alpha=1}^{k} c_{\alpha} f_{\alpha}(x) \geqslant \varepsilon|x|^{2}$; therefore the cone $K$ is contained in a closed, convex cone in $\mathbf{R}^{k}$ that is nondegenerate, in the sense that it contains no complete straight line.

Assume now, for the moment, that $n<\infty$ and suppose that the conclusion fails: since the image cone $K=F\left(\mathbf{R}^{n}\right)$ is closed and not convex, there must exist a ray $\Lambda=\{\lambda \eta\}_{\lambda>0}, \eta=\left(\eta_{1}, \eta_{2}, \eta_{3}\right)$, which is outside of $K$ but interior to its convex hull; therefore for each plane $\Pi$ containing $\Lambda$ there are points of $K$ that lie on each side of $\Pi$. Let two such planes be defined by $\Sigma_{\alpha=1}^{3} a_{\alpha} u_{\alpha}=0$ and $\sum_{\alpha=1}^{3} b_{\alpha} u_{\alpha}=0$, that is to say, assume that $(a)=\left(a_{1}, a_{2}, a_{3}\right)$ and $(b)=\left(b_{1}, b_{2}, b_{3}\right)$ are linearly independent solutions of the equation with unknowns $u_{1}, u_{2}, u_{3}$,

$$
\sum_{\alpha=1}^{3} \eta_{\alpha} u_{\alpha}=0
$$

then $(a)$ and $(b)$ are a basis for all the solutions of (2) and therefore, for any pair of real numbers $(\lambda, \mu) \neq(0,0)$ the quadratic form

$$
g(x)=\sum_{\alpha=1}^{3}\left(\lambda a_{\alpha}+\mu b_{\alpha}\right) f_{\alpha}(x)
$$

vanishes precisely at the points $x \in \mathbf{R}^{n}$ such that $F(x)=\left(f_{1}(x), f_{2}(x), f_{3}(x)\right)$ lies in the plane

$$
\left\{(u)=\left(u_{1}, u_{2}, u_{3}\right) \in \mathbf{R}^{3} \mid \sum_{\alpha=1}^{3}\left(\lambda a_{\alpha}+\mu b_{\alpha}\right) u_{\alpha}=0\right\}
$$

passing through the half-line $\Lambda$ generated by $\left(\eta_{1}, \eta_{2}, \eta_{3}\right)$.

On the one hand for each pair $(\lambda, \mu)$ the plane (4) admits points of $K$ on each of its two sides; therefore the quadratic form $g(x)$ in (3) is indefinite for each $(\lambda, \mu)$. On the other hand the complete line generated by $\Lambda$ meets the image cone $K$ only at the origin, since $\Lambda \cap K=\{0\}$ and $K \backslash\{0\}$ is contained in an open half-space, while $-\Lambda$ is in the opposite half-space. Thus the two quadratic forms $\sum_{\alpha=1}^{3} a_{\alpha} f_{\alpha}(x)$, and $\Sigma_{\alpha=1}^{3} b_{\alpha} f_{\alpha}(x)$ have no common zeros except $x=0$. This contradicts the main theorem of [1], see also [2] according to which, if two quadratic forms on $\mathbf{R}^{n}$, for $n \neq 2, n<\infty$ have only 0 as a common null-vector, then there is a linear combination of them that is positive definite. Therefore, if $n<\infty$, we have proved that the image cone must be convex.

In the case of an infinite dimensional linear space $\mathbf{R}^{\infty}$, with any topological structure, the same conclusion holds, since the image cone under $F$ of any finite dimensional vector subspace is convex. The theorem is thereby proved.

The following two examples show the futility of attempts to prove certain naive generalizations of the theorem. 
(A) Consider in $\mathbf{R}^{n}(3<n<\infty)$ the three quadratic forms

$$
f_{1}(x)=\left(x_{1}\right)^{2}, \quad f_{2}(x)=x_{1} x_{2}, f_{3}(x)=\left(x_{2}^{2}\right)-\sum_{j=3}^{n}\left(x_{j}\right)^{2}:
$$

the quadratic form $f_{1}$ is positive semidefinite (but not definite); yet the image cone under the map $F=\left(f_{1}, f_{2}, f_{3}\right): \mathbf{R}^{n} \rightarrow \mathbf{R}^{3}$ is the set

$$
\left\{(u) \mid u_{1}>0, u_{1} u_{3} \leqslant\left(u_{2}\right)^{2}\right\} \cup\left\{(u) \mid u_{1}=u_{2}=0\right\},
$$

which is not convex.

(B) Consider in $\mathbf{R}^{n}(3 \leqslant n<\infty)$ the four quadratic forms

$$
\begin{aligned}
& f_{1}(x)=\left(x_{1}\right)^{2}+\sum_{j=3}^{n}\left(x_{j}\right)^{2}, \quad f_{2}(x)=\left(x_{1}\right)^{2}-\left(x_{2}\right)^{2}, \\
& f_{3}(x)=2 x_{1} x_{2}, \quad f_{4}(x)=x_{2}^{2}-\sum_{j=3}^{n}\left(x_{j}\right)^{2}:
\end{aligned}
$$

the quadratic form $2 f_{1}+f_{4}$ is positive definite; yet the image cone $F\left(\mathbf{R}^{n}\right) \subset \mathbf{R}^{4}$ under the map $F=\left(f_{1}, f_{2}, f_{3}, f_{4}\right)$ is the 3-dimensional semi-algebraic set

$$
\left\{(u)=\left(u_{1}, u_{2}, u_{3}, u_{4}\right)\left|u_{1}+u_{4}=\sqrt{\left(u_{2}\right)^{2}+\left(u_{3}\right)^{2}}, u_{1}>\right| u_{2}+u_{4}\left|, u_{1}>u_{2}+\right| u_{4} \mid\right\}
$$

which is not convex.

We conclude with an application. Let $\mathcal{H}$ be any linear function space, in which three quadratic forms are given. For instance, let $\mathcal{H}$ be the function space on an open Riemannian manifold consisting of all real-valued functions $\phi$ that are square-summable, along with their first and second derivatives; let $f_{\alpha}(\phi)(\alpha=$ $1,2,3)$ denote respectively the squared $L_{2}$-norms $\|\phi\|^{2},\|\nabla \phi\|^{2},\|\Delta \phi\|^{2}$ of $\phi$, of its gradient, and of its Laplacian, with respect to the Riemannian metric, for any $\phi \in \mathcal{H}$. It is known that $f_{2}(\phi)$ has an upper bound in terms of $f_{1}(\phi)$ and $f_{3}(\phi)$, that $f_{1}$ has a positive lower bound in terms of $f_{2}(\phi)$ and $f_{3}(\phi)$, and that $f_{3}$ has a positive lower bound in terms of $f_{1}(\phi)$ and $f_{2}(\phi)$ (if $f_{2}(\phi)$ is sufficiently large); however it is not easy to determine in general what the best estimates are, nor what other constraints there are (if any) on the possible values of $\left(f_{1}(\phi), f_{2}(\phi), f_{3}(\phi)\right) \in \mathbf{R}^{3}$. The main theorem of this paper proves that the set of possible values of these triple functionals form a convex cone $K$ in $\mathbf{R}^{3}$; from this one easily deduces that the set of values of $\left(f_{1}, f_{2}, f_{3}\right)$ is not enlarged if we replace real-valued functions with complex-valued ones (or $\mathbf{R}^{k}$-valued ones for any euclidean $\mathbf{R}^{k}$ ). Finally, since the image cone $K$ is convex, it is completely determined by its dual cone $K^{*}$ consisting of all the triples $\left(v_{1}, v_{2}, v_{3}\right) \in \mathbf{R}^{3}$ such that, for all $\phi \in \mathcal{H}, \Sigma_{\alpha=1}^{3} v_{\alpha} f_{\alpha}(\phi)>0$. This reduces the question to the apparently simpler one of determining the set of all triples $\left(v_{1}, v_{2}, v_{3}\right)$ for which a certain selfadjoint completion of the differential operator

$$
\phi \rightarrow v_{3} \Delta^{2} \phi-v_{2} \Delta \phi+v_{1} \phi
$$

(determined by asymptotic conditions of $\phi$ at "infinity", or by boundary conditions if the manifold is a compact manifold with boundary) is positive semidefinite. 
This last problem is far from trivial even in one of the simplest conceivable cases, that of functions $\phi$ on an open interval $[0, a] \subset \mathbf{R}$; let

$$
f_{1}(\phi)=\frac{1}{a} \int_{0}^{a}|\phi(t)|^{2} d t, \quad f_{2}(\phi)=a \int_{0}^{a}\left|\phi^{\prime}(t)\right|^{2} d t, \quad f_{3}(\phi)=a^{3} \int_{0}^{a}\left|\phi^{\prime \prime}(t)\right|^{2} d t
$$

(note that the coefficients are chosen so that the functionals $f_{\alpha}$ are independent of a). In this case it has been determined that the image cone of $\mathcal{C}$ under $\left(f_{1}, f_{2}, f_{3}\right)$ is bounded by the convex hull of an infinite sequence of analytic, convex, conical surfaces, each one obtained by solving a certain one-parameter family of fourth order, ordinary differential equations with appropriate (but unusual) boundary conditions.

\section{REFERENCES}

1. E. Calabi, Linear systems of real quadratic forms, Proc. Amer. Math. Soc. 15 (1964), 844-846.

2. P. Finsler, Über das Vorkommen definiter und semidefiniter Formen in Sharen quadratischer Formen, Comment. Math. Helv. 9 (1937), 188-192.

Department of Mathematics, University of Pennsylvania, Philadelphia, Pennsylvania 19104 